\title{
Extent of Microbial Contamination of Refined and Unrefined Vegetable oils sold in South-west Nigeria
}

\author{
Oluwafemi Flora $^{1 *}$, Oni Eniola Oluyemisi ${ }^{1}$, Kareem Sarafadeen Olateju ${ }^{1}$, \\ Omemu Adebukunola Mobolaji ${ }^{1}$, Kolapo Lawrence Adelodun ${ }^{2}$
}

${ }^{1}$ Department of Microbiology, Federal University of Agriculture, Abeokuta, Nigeria

${ }^{2}$ Department of Biological Sciences, Augustine University, Epe, Nigeria

A R T I C LE I N F O

\section{Research Article}

Received 11 December 2017

Accepted 27 January 2018

Keywords:

Refined vegetable oil

Unrefined vegetable oil

Mycotoxigenic fungi

Contamination

Oil consumption

*Corresponding Author:

E-mail: oni_eniola51@yahoo.com

A B S T R A C T

Oils constitute a major source of plant-based protein. A major limitation to optimal oil consumption in sub-tropical region is fungal infestation and consequent mycotoxin contamination. Ten refined and eight unrefined vegetable oils were randomly purchase from open markets and screened for microbial contamination using standard microbial procedures. Twenty six fungi isolates were obtained from the vegetable oil samples, the isolates were identified as Aspergillus fumigatus (43.0\%), Mucor (17.9\%), Saccharomyces cerevisiae (10.7\%), Aspergillus niger (7.1\%), Aspergillus flavus (7.1\%), Penicillium spp (7.1\%), Aspergillus oryzae (3.6\%), Mucor (17.9\%) and Rhizopus spp $(3.6 \%)$. Five out of the ten refined vegetable oil samples had no fungal contamination. $A$. flavus and $A$. oryzae were absent in all the refined oil samples while $A$. niger was absent in all the unrefined oil samples. Isolation of mycotoxigenic fungi such as Aspergillus spp. is of vital importance in the food industry. Education and training of processors and consumers is recommended.

DOI: https://doi.org/10.24925/turjaf.v6i4.396-400.1430

\section{Introduction}

Agricultural products such as oils make an excellent substrate for the growth of mould, fungus and other microbiological forms. As these moulds grow and derive their cell carbon from these oils, they cause deterioration in oil quality by producing free fatty acids under local storage conditions. It has been reported that most tropical edible oils are heavily infested by moulds (Manonmani et al., 2005).

Moulds are part of the natural environment, they are the most typical form of fungus on earth and comprise approximately $25 \%$ of the earth biomass. Apart from moulds capable of digesting and exhausting nutrient content of crops, more to be feared is the added danger of aflatoxin production in the oils (Tagoe, 2008).

Mycotoxins are considered an important problem throughout the world in terms of public health, agriculture and economics. They are natural poisons produced by fungi as secondary metabolites (Baskaya et al., 2006). Three genera are responsible for the majority of the mycotoxins with which FDA is concerned: The Aspergillus spp Penicillium spp, and Fusarium spp.
The term mycotoxin is derived from the greek word "mykes" meaning fungus and the latin word "toxicum" meaning poison. Accumulation of mycotoxins in food and feeds represent a major threat to human and animal health Mupunga et al., (2014) and Oluwafemi and Taiwo (2004).

Aflatoxins are secondary metabolite of mycotoxin produced by the fungi Aspergillus flavus and Aspergillus parasiticus which commonly infect food produce such as maize, oilseed, peanut and tree nuts (Mmongoyo et al., 2017)

Contamination of food with aflatoxin is more prevalent in tropical and sub tropical areas where environmental conditions such as high temperature and humidity prevail (Klich, 2007). Many vegetable oils are consumed directly or used as ingredients in food and they are primarily from seeds of oilseed plants (Behrman and Venkat, 2005).

Vegetable oils are majorly used for cooking, processing in the food industry and meeting dietary demands. Often times they are contaminated by mycotoxins and heavy metals (Ma et al., 2015). 
Refined oils are purified oils obtained from oil cakes using a process of solvent extraction. Refined cooking oils are made by highly intensive mechanical and chemical (solvent extraction) process to extract the oil from the seeds and vegetable products. The crushed seeds are heated to a temperature between $110^{\circ} \mathrm{C}-180^{\circ} \mathrm{C}$ in a steam bath to start the oil extraction process.

However, unrefined oils are obtained by a process of pressing the seeds or other vegetable materials. High level of mould in oil seed and their products are undesirable due to their capability of digesting and exhausting the nutrient contents of crops as well as the added danger of aflatoxins in the oils. In view of this assertion, this study assessed the quality of marketed vegetable oils in Southwest Nigeria.

\section{Materials and Methods}

\section{Sample Collection}

Eight (8) samples of unrefined oils were collected from vegetable oil factories in Lagos, Ibadan and Abeokuta. The samples were aseptically collected in sterile glass bottles, capped and labelled properly and transferred immediately to the laboratory for analysis.

Ten (10) brands of vegetable oils (refined) were also purchased from various markets in Lagos, Ibadan and Abeokuta and taken to the laboratory for further analysis.

\section{Culturing of Refined and Unrefined Oil Samples}

Serial dilution of homogenized oil was done, by pipetting $1 \mathrm{ml}$ of oil into a test tube containing $9 \mathrm{ml}$ of peptone water and $1 \mathrm{ml}$ was pippeted again from the 10 $\mathrm{ml}$ that was made up to another test tube containing $9 \mathrm{ml}$ of peptone water and was done in 10 folds. The $10^{3}-10^{5}$ dilutions were plated on Sabouraud Dextrose Agar (SDA) at $28^{\circ} \mathrm{C}$ in an incubator for five days. Each dilution was replicated five times and the fungal counts estimated in colony forming unit/gram (CFU/g) of samples.

\section{Identification of Fungal Isolates}

Fungal isolates were identified based on morphological and cultural characteristics described by Barnet et al. (2003). Additional characterization tests were carried out according to standard methods of Tsuneo (2010) and Klich (2002).

\section{Results}

Identification and Characterization of Fungi Isolates from The Oil Samples

Table 1 shows the identification of unrefined vegetable oil fungal isolates. The characteristics of the mould isolated were yellow to brown, green filamentous colonies, smooth walled conidiophores to budding yeast cells. It revealed that twelve mould were isolated of which Aspergillus fumigatus (8) had the highest occurring percentage, followed by A. flavus (2), A. oryzae (1) and Saccharomyces cerevisae (1).

Table 2 shows the identification of refined vegetable oil fungal isolates. It revealed that fourteen fungi were isolated and identified as Rhizopus spp. (1), Mucor spp. (4), A. niger (2), A. fumigatus (3), Penicillium spp. (2) and Saccharomyces cerevisae (2). All the yeasts identified were budding yeast. Moulds included hyphae without rhizoids and large conidiophores. The colour of the colonies included creamy-white, bluish-green, green, brownish-black and green filamentous colonies.

\section{Percentage of Fungal Genera in The Oil Samples}

Table 3 shows the frequency of occurrence $(\%)$ of fungi in oil samples. Eight fungi isolated from refined oil samples were Aspergillus fumigatus (43.0\%), Mucor spp (17.9\%), S. cerevisae (10.7\%), A. niger, A. flavus and Penicillium spp (7.1\%) while A. oryzae and Rhizopus spp are $3.6 \%$ respectively.

\section{Distribution of Fungi on Refined and Unrefined} Vegetable Oil Samples

Table 4 presents the distribution of fungal isolates in the refined vegetable oil samples studied. Five (Cotton, Canola, Sunflower, Soya and Palm oil) out of the ten refined vegetable oil samples analysed had no fungal contamination. Of the eight mould identified, A. flavus and $A$. oryzae were absent in all the refined oil samples. $A$ fumigatus was found only in corn oil and palm kernel oil, $S$. cerevisae occurred only in coconut oil while Mucor spp was present in both corn oil and olive oil. Rhizopus spp. and A. niger were present in groundnut oil while Penicilliun notatum was present in palm kernel oil only.

Table 1 Characteristics of fungal isolates of unrefined vegetable oil

\begin{tabular}{c|llll}
\hline S/N & Isolate code & Macroscopy & Microscopy & Organism \\
\hline 1 & UR1 & Brownish filamentous colonies & Smooth walled conidiophores & Aspergillusfumigatus \\
2 & UR2 & Brownish filamentous colonies & Smooth walled conidiophores & $\begin{array}{l}\text { Aspergillusfumigatus } \\
\text { (a) Aspergillusoryzae }\end{array}$ \\
3 & UR1 & (a)Brownish filamentous colonies & (a)Slender conidiphore & (b) Mucor sp. \\
4 & UR3 & (b) White cotton-like colonies & (b) Hyphae without rhiziod sp. & (b) \\
5 & UR4 & Brownish filamentous colonies & Smooth walled conidiophores & Aspergillusfumigatus \\
6 & UR5 & Brownish filamentous colonies & Smooth walled conidiophores & Aspergillusfumigatus \\
7 & UR6 & Green filamentous colonies & Smooth walled conidiophores & Aspergillusfumigatus \\
8 & UR7 & Green filamentous colonies & Smooth walled conidiphores & Aspergillusfumigatus \\
9 & UR8 & Green filamentous colonies & Smooth walled conidiophores & Aspergillusfumigatus \\
10 & UR4 & Creamy white colonies & Budding yeast cell & Saccharomyces cerevisiae \\
11 & UR6 & Yellowish filamentous colonies & Globose conidiophores & Aspergillusflavus \\
12 & UR3 & Yellowish filamentous colonies & Globose conidiophores & Aspergillusflavus \\
\hline UR1 Unrefined mat 1-Soya extract; UR2- Unrefined mat 2-Palm stearin; UR3- Unrefined Raw mat 3-Palm kernel; UR4- Unrefined Raw mat 4- \\
Soya extract; UR5 - Unrefined mat 5-Palm olein; UR6- Unrefined mat 6-Palm oil; UR7 -- Unrefined mat 7-Palm oil; UR8 -- Unrefined mat 8- \\
Palm oil
\end{tabular}


Table 2 Characteristics of fungal isolates of refined vegetable oil

\begin{tabular}{c|llll}
\hline S/N & Isolate names & Macroscopy & Microscopy & Organism \\
\hline 1 & Groundnut oil & Cotton-like colonies & Hyphae with rhizoids & Rhizopus spp. \\
2 & Groundnut oil & Cotton-like colonies & Hyphae without rhizoids & Mucor spp \\
3 & Cotton oil & Creamy white colonies & Budding yeast cell & Saccharomyces cerevisiae \\
4 & Palm oil & Cotton-like colonies & Hyphae without rhizoids & Mucor spp \\
5 & Olive oil & Cotton-like colonies & Hyphae without rhizoids & Mucor spp \\
6 & Corn oil & Cotton-like colonies & Hyphae without rhizoids & Mucor spp \\
7 & Groundnut oil & Black filamentous colonies & Large/globoseconidiphores & Aspergillus niger \\
8 & Corn oil & Green filamentous colonies & Smooth walled conidiophores & Aspergillus fumigatus \\
9 & Corn oil & Green filamentous colonies & Smooth walled conidiophores & Aspergillus fumigatus \\
10 & Palm kernel oil & Green filamentous colonies & Smooth walled conidiphores & Aspergillus fumigatus \\
11 & Palm kernel oil & White filamentous colonies & Large conidiphores & Penicillium spp \\
12 & Palm oil & Whitefilamentouscolonies & Large conidiphores & Penicillium spp \\
13 & Corn oil & Black filamentous colonies & Large/globoseconidiphores & Aspergillus niger \\
14 & Olive oil & Creamy white colonies & Budding yeast cell & Saccharomyces cerevisiae \\
\hline
\end{tabular}

Table 3 Frequency of occurrence $(\%)$ of fungi in oil samples

\begin{tabular}{l|cc}
\hline \multicolumn{1}{c|}{ Fungal isolates } & Frequency & Percentage \\
\hline Aspergillus fumigates & 12 & 43 \\
Aspergillus niger & 2 & 7.1 \\
Aspergillus oryzae & 1 & 3.6 \\
Aspergillus flavus & 2 & 7.1 \\
Mucor spp & 5 & 17.9 \\
Penicillium spp. & 2 & 7.1 \\
Rhizopus spp. & 1 & 3.6 \\
Saccharomyces cerevisae & 3 & 10.7 \\
\hline
\end{tabular}

Table 4 Distribution of fungal isolates in the refined vegetable oil samples

\begin{tabular}{l|cccccccccc}
\hline \multicolumn{1}{c|}{ Isolates } & COT & SUN & CAN & COR & COC & OLI & SOY & PALK & PAL & GRO \\
\hline Aspergillus fumigatus & - & - & - & + & - & - & - & + & - & - \\
A. flavus & - & - & - & - & - & - & - & - & - & - \\
A. oryzae & - & - & - & - & - & - & - & - & - & - \\
Sacharomyces Cerevisae & - & - & - & - & ++ & - & - & - & - & - \\
Mucor spp & - & - & - & + & - & ++ & - & - & - & - \\
Penicillium notatum & - & - & - & - & - & - & - & + & - & - \\
Rhizopus spp & - & - & - & - & - & - & - & - & - & + \\
A. niger & - & - & - & - & - & - & - & - & - & ++ \\
\hline
\end{tabular}

$+=$ One occurrence; $++=$ Two occurrence; $-=$ No occurrence; COT: Cotton oil, SUN: Sunflower oil, CAN: Canola oil, COR: Corn oil, COC:

Coconut oil, OLI: Olive oil, SOY: Soya oil, PALK: Palm kernel oil, PAL: Palm oil, GRO: Groundnut oil

Table 5 Distribution of fungal isolates in the unrefined vegetable oils from oil factories

\begin{tabular}{l|cccccccc}
\hline \multicolumn{1}{c|}{ Isolates } & $\mathrm{A}$ & $\mathrm{B}$ & $\mathrm{C}$ & $\mathrm{D}$ & $\mathrm{E}$ & $\mathrm{F}$ & $\mathrm{G}$ & $\mathrm{H}$ \\
\hline Aspergillus fumigatus & ++++ & + & - & - & - & + & + & + \\
A. flavus & - & - & + & + & - & - & - & - \\
A. oryzae & - & - & + & - & - & - & - & - \\
A. niger & - & - & - & - & - & + & - & - \\
Sacharomyce cerevisae & + & - & - & - & - & - & - & - \\
Mucorspp & - & - & ++ & - & - & - & - & - \\
Penicillium spp & - & - & - & + & - & - & - & - \\
Rhizopus spp & - & - & - & - & - & - & - & - \\
\hline
\end{tabular}

$+=$ One occurrence, $++=$ Two occurrence, $++++=$ Four occurrence, - = No occurrence, A - Unrefined mat 1-Soya extract, B - Unrefined mat 2Palm stearin, C - Unrefined mat 3-Palm kernel, D - Unrefined mat 4-Soya extract, E - Unrefined mat 5-Palm olein, F- Unrefined mat 6-Palm oil,

$\mathrm{G}$ - Unrefined mat 7-Palm oil, $\mathrm{H}$ - Unrefined mat 8- Palm oil

Table 5 presents the distribution of fungal isolates in the unrefined vegetable oil samples studied. A. fumigatus was present in unrefined oil samples $\mathrm{C}, \mathrm{D}$ and $\mathrm{E}$ while $A$. flavus was present only in samples C and D. A. oryzae, $S$. cerevisae and Penicillium spp. were present in only A, C and D unrefined oils respectively while Rhizopus spp and A. niger were absent in all the oil samples.

\section{Discussion}

It has been estimated by the Food and Agriculture Organization (2002) that $25 \%$ of the world's crops are affected by mycotoxins produced by moulds. It is one of the most potent naturally occuring mutagens and carcinogens known. Global view of aflatoxin- 
contaminated food items revealed that virtually all foods are vulnerable including edible oil seeds.

In agreement with Umeh et al., (2000) and Bankole et al., (2004), the present study showed that members of Aspergillus spp. was highly prevalent. Aspergillus isolated from the vegetable oils has been known to produce aflatoxin $\mathrm{B}_{1}, \mathrm{~B}_{2}, \mathrm{G}_{1}$ and $\mathrm{G}_{2}$, (CDC, 2006).

Ragab et al. (2001) had reported that mycotoxins mainly formed by certain filamentous fungi belonging to the genera Aspergillus, Penicillium, Alternaria and Fusarium species, may grow on a number of food commodities which are the major contributors of food spoilage. The high frequency and abundance of Aspergillus spp. in the findings could be due to failures during food production and storage.

The results also agrees with the findings of IARC (2002) that maize, corn, cotton seeds, oil seed and most crops are frequently contaminated with aflatoxin. It is also in line with the work of Ngoko et al. (2001) who reported that Fusarium and Aspergillus were the most prevalent fungi on the sampled commodities with isolation frequency varying from 20 to $100 \%$.

This work has been able to establish that unrefined oil has more fungal load than the refined oils which are in accordance with the findings of Elzupit et al. (2010) and Mariod and Idris (2015) and that consumers should limit the rate at which they consume unrefined oils because they are more susceptible to aflatoxins contamination. Hence, protective measures must be followed during the refinning processes.

It could be deduced that the significant amount of impurities obtained in this oils could be linked to the method of storage. These storage facilities are mostly metallic and thus rust with time, thereby releasing toxic chemicals into the oil and this is in accordance with the findings of Odoh et al. (2017).

This work has been able to establish that refining reduces aflatoxin level in vegetable oil which corroborates the findings of Banu (2004).

Elzupir and Abdulaziz (2014) recommended keeping oil seed at freezing temperature until production will reduce or arrest proliferation of mould invariably aflatoxin thereby ensuring food safety.

Technologies such as biological control, improved packaging, irradiation and mechanism of ozone to inhibit microbial populations in food via the progressive oxidation of vital cellular components can also minimise aflatoxigenic mould contamination in agricultural products Udomkun et al. (2017).

\section{Conclusion}

Fungal contamination rate should not be neglected. Hence, isolation of mycotoxigenic fungi such as Aspergillus species is of vital importance in the food industry therefore, it is feasible to decrease fungal contamination by sufficient education in the field of food industry. Regulatory agencies in Nigeria should equally monitor mould growth in oils meant for human consumption to avoid food poisoning and contamination.

\section{Acknowledgement}

Oni E. acknowledges the Mycotoxin Laboratotory of the National Agency for Food Drug Administration and Control (NAFDAC) for providing equipments for this research work.

\section{References}

Bankole SA, Ogunsanwo BM, Mabekoje OO. 2004. Natural occurrence of moulds and aflatoxin B1 in melon seeds from markets in Nigeria. Food and Chemical Toxicology 42: 1309-1314.

Banu N. 2004. Studies on mycobiota from sunflower oil refineries with reference to mycotoxins from the fungi and the feed and oil samples. Ph.D. Thesis, University of Madras, Chennai.

Barnett IA. 2003. Beginnings of Microbiology and Biochemistry. The contribution of yeast Research. Microbiology 149: 557-567.

Baskaya R, Aydin A, Yildz A, Bostank B. 2006. Aflatoxin M1 levels of some cheese variety in Turkey. Journal of Animal Science 62: 778-780.

Behrman E, Venkat G. 2005, Cholesterol and plants. Journal of Chemical Education 82: 1790-1793.

CDC. 2006. Center for Disease Control, Atlanta Georgia. Outbreak of Aflatoxicosis in some parts of Africa". 97(5): 346-352

Elzupir AO, Suliman, MA, Ibrahim IA, Fadul MH, Elhussein AM. 2010. Aflatoxins levels in vegetable oils in Khartoum State, Sudan. Mycotox. Res, 26: 69-73.

Elzupir AO, Abdulaziz SA. 2014. Quantitative cancer risk of aflatoxin in peanut butter and vegetable oils: Sudan case study. Toxin Reviews 33.4: 202-205.

FAO/WHO. 2002. Standard Programme of Codex Alimentarius Commission.

IARC. 2002. International Agency for Research on Cancer). 2002. World health organization international agency for research on cancer. Aflatoxins: B1, B2, G1, G2, M1. In: Some traditional herbal medicines, some mycotoxins, naphthalene and styrene. IARC monographs on the evaluation of carcinogenic risks to humans, Lyon, France 82: 171-175.

Klich MA. 2002. Identification of common Aspergillus species. Central bureauvoorschimmel cultures, Utrecht, the Netherlands, pp $1-116$.

Klich MA. 2007. Aspergillus flavus: the major producer of aflatoxin. Molecular Plant Pathology 8(6): 713-722.

Ma F, Wu R, Li P, Yu L. 2015. Analytical approaches for measuring pesticides, mycotoxins and heavy metals in vegetable oils: A review. European journal of lipid science and technology, 118 (3): 339-352.

Mariod AA, Idris YMA. 2015. Aflatoxin B1 levels in groundnut and sunflower oils in different Sudanese states. Food Additives \& Contaminants: Part B, (4): 266-270.

Manonmani HK, Anand S, Chandrashekar A, Rati ER. 2005. Detection of aflatoxigenic fungi in selected food commodities by PCR. Process Biochemistry 40: 2859-2864.

Mmongoyo JA, Wu F, Linz JE, Nair MG, Mugula JK, Tempelman RJ, Strasburg GM. 2017. Aflatoxin levels in sunflower seeds and cakes collected from micro-and small-scale sunflower oil processors in Tanzania. PloS one, 12:4

Munpuga I, Lebelo SL, Mngqawa P, Rheeder JP, Katerere DR. 2014. Natural occurrence of aflatoxins in peanuts and peanut butter from Bulawayo, Zimbabwe. J Food Prot. 77 (10): 1814-8

Ngoko Z, Marasas WFO, Rheeder JP, Sherphard GS, Wingfield MJ. 2001. Fungal infection and mycotoxin contamination of maize in the humid forest and the Western highlands of Cameroon. Phytoparasitica 29(4): 352-360.

Odoh CK, Amapu TY, Orjiakor IP, Martins PE, Seibai BT, Akpi U. K, Nwankwegu AS. 2017. Assessment of mold contamination and physicochemical properties of crude palm oil sold in Jos, Nigeria. Food science \& nutrition, 2: 310-316.

Oluwafemi F, Taiwo VO 2004. Reversal to toxigenic effects of aflatoxin $\mathrm{B}_{1}$ on cockerels by alcoholic extract of African nutmeg, Monodoramyristica. Journal of the Science of Food and Agriculture 84:330-340. 
Tagoe SMA. 2008. Effect of mycotoxigenic microorganisms on palm fruits and palm oil produced at the cottage industry level. Nottingham University, United Kingdom, PhD Thesis.

Tsuneo W. 2010. Pictorial atlas of soil and seed fungi: Morphologies of cultural fungi and key to species. Third edition. CRC press.
Umeh VC, Waliyar F, Traoré A. 2000. Aspergillus species colonization of termite-damaged peanuts in parts of West Africa and its control prospects. Peanut Science 27(1): 1-6.

Udomkun P, Wiredu AN, Nagle M, Müller J, Van B, Bandyopadhyay R. 2017. Innovative technologies to manage aflatoxins in foods and feeds and the profitability of application. Food Control 76: 127-138 